\title{
MicrostruCtURe of MS1 MARAging STEel in 3D-PRinTED Products AFTer Semi-Solid Processing
}

\author{
Kateřina Rubešová, Štěpán Jeníček, Josef Káňa \& Ivana Zetková
}
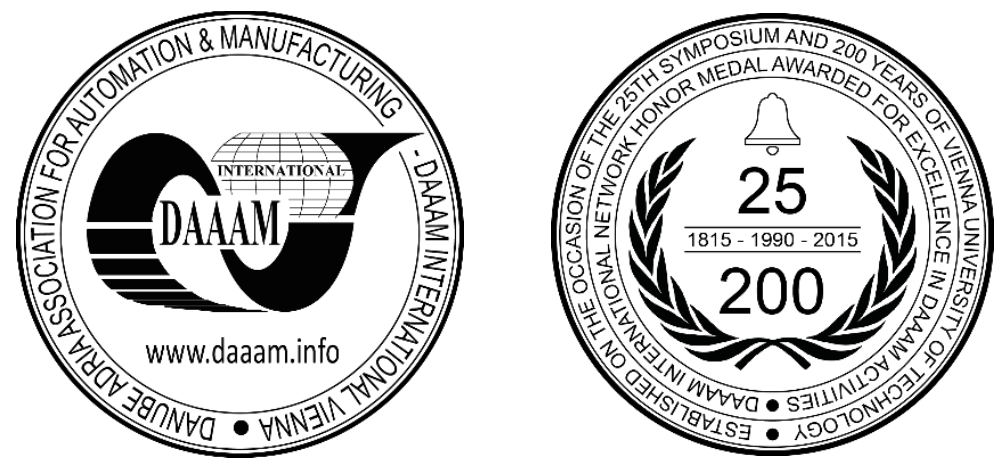

This Publication has to be referred as: Rubesova, K[aterina]; Jenicek, S[tepan]; Kana, J[osef] \& Zetkova, I[vana] (2016). Microstructure of MS1 Maraging Steel in 3D-Printed Products After Semi-Solid Processing, Proceedings of the 27th DAAAM International Symposium, pp.0467-0472, B. Katalinic (Ed.), Published by DAAAM International, ISBN 978-3-902734-08-2, ISSN 1726-9679, Vienna, Austria

DOI: $10.2507 / 27$ th.daaam.proceedings.070

\begin{abstract}
The importance of 3D printing in today's industry is continuously increasing. One of the materials which are frequently used in this process is the precipitation-hardenable MS1 Maraging steel whose properties after conventional treatment have been thoroughly mapped. Maraging steels belong to the strongest homogeneous steel materials ever. Their alloying additions include cobalt, molybdenum, titanium and aluminium, and their unusual properties make them candidates for heavy-duty parts operating predominantly under dynamic loading. Semi-solid processing of this particular steel enables near-net shape products to be made using a single production step. Furthermore, unconventional microstructure and physical, chemical and mechanical properties can be obtained by this process. The semi-solid processing route and the mechanical properties achieved by it can expand the potential uses of this material. Semi-solid processing of small volumes of material has already been mastered with high-alloy steels which offer a wide range of forming temperatures. However, as this process is technically demanding, its use for medium and low-alloy ultra-high-strength steels has not been explored in greater detail yet. In the present experiment, MS1 Maraging steel was studied. The experimental feedstock was made by 3D printing. The initial set of semi-solid processing parameters was proposed on the basis of calculation and then tested experimentally. In this article, description of the microstructures of this steel before and after several semi-solid processing routes is given, based on examinations using optical and electron microscopy.
\end{abstract}

Keywords: Key words: semi-solid processing; rapid solidification; near net shape; maraging steel; 3D printing

\section{Introduction}

Although the principle of thixoforming has been known since the early 1970s, the process is still ranked among unconventional metalworking methods. It relies on the thixotropic behaviour which occurs in materials heated into the semi-solid state, i.e. to a temperature between their solidus and liquidus. Thixoforming belongs to precision casting and forming methods. During the forming process, shear forces act on the partially melted material and cause its viscosity to decrease significantly. This effect is known as thixotropy [1]. The liquid fraction in the workpiece being formed is normally between 10 and $60 \%$ [2]. The technique was initially developed mainly for low-melting materials: aluminium, magnesium and tin alloys [3]. Thanks to intensive research, commercial machines for the thixoforming of low-melting alloys are already (though scarcely) in use. Yet, when it comes to the thixoforming of steels, basic research is lagging 
behind due to technological complexity of the issue. It is unfortunate, considering the vast potential of this technology. Nevertheless, semi-solid forming enables difficult-to-form materials to be worked by means of a single forming operation. In addition, more complex and intricate shapes can be produced by this technique than

by conventional forming routes. In addition, unconventional microstructures can be produced by thixoforming even in ordinary materials.

The research into the use of steels has made less progress than with low-melting alloys because it faced substantial technological difficulties. Among them are the high process temperatures, narrow temperature intervals, and the ensuing need for strict control of the temperature field [4]. The only detailed description of this process available so far in literature concerns high-alloy steels. Up to now, no comprehensive research into the behaviour of low-alloy medium-carbon and low-carbon steels has been undertaken - which is the focus of the present experiment.

Its objective was to explore the capabilities of mini-thixoforming of 3D-printed steel workpieces, and obtain the desired type of microstructure by the process. Mini-thixoforming enables near-net-shape products to be obtained. These are geometrically accurate and require only minimal subsequent finishing for the final surface quality and geometric accuracy, unlike the conventional formed products. Mini-thixoforming therefore offers material, energy, and financial savings.

\section{Experimental programme}

For this investigation, the low-carbon MS1 tool steel in the form of 3D-printed feedstock was chosen as the experimental material (Tab. 1). It is one of the popular materials for 3D printing. It is a precipitation-hardenable steel whose properties after conventional treatment have been thoroughly mapped. Maraging steels belong to the strongest homogeneous steel materials ever. They are alloyed with cobalt, molybdenum, titanium, and aluminium. Their unusual properties make them candidates for heavy-duty parts operating predominantly under dynamic loading. Their semi-solid processing parameters window is very narrow due to their chemical composition. This makes the choice of suitable thixoforming temperature all the more difficult.

\begin{tabular}{|l|l|l|l|l|l|l|l|l|l|}
\hline $\mathrm{Ni}$ & $\mathrm{Co}$ & $\mathrm{Mo}$ & $\mathrm{Ti}$ & $\mathrm{Al}$ & $\mathrm{Cr}, \mathrm{Cu}$ & $\mathrm{C}$ & $\mathrm{Mn}, \mathrm{Si}$ & $\mathrm{P}, \mathrm{S}$ & $\mathrm{Fe}$ \\
\hline $17-19$ & $8.5-9.5$ & $4.5-5.2$ & $0.6-0.8$ & $\begin{array}{c}0.05- \\
0.015\end{array}$ & $\leq 0.5$ & $\leq 0.3$ & $\leq 0.1$ & $\leq 0.01$ & balance \\
\hline
\end{tabular}

Table 1. Chemical composition of the MS1 Maraging steel measured by chemical analysis

In order to identify suitable thixoforming parameters, the physical and chemical properties of the material and their profiles throughout the entire heating process must be known. One of the key characteristics relevant to semi-solid processing is the width of the interval between the solidus and liquidus. The interval recommended specifically for minithixoforming is 10-30\% [5,6]. Finding these parameters experimentally is time-consuming. In this case, the first step involved calculations which were completed using the JMatPro software. As the chemistry of this steel is extremely complex, the results should be considered with caution, not to mention the fact that the actual process is very rapid. The calculated theoretical onset of melting is at $1395^{\circ} \mathrm{C}$. The material becomes completely melted at $1445^{\circ} \mathrm{C}$ (Fig. 1). Hence, the freezing range width is approximately $50^{\circ} \mathrm{C}$. According to the calculation, the theoretical interval that is usable for semi-solid forming is $1408-1420^{\circ} \mathrm{C}$. The advantage of this material is that its melting curve is smooth without any major curvature. This is favourable for the temperature field control. The narrow processing parameters window and the high temperature pose major difficulties for the process.

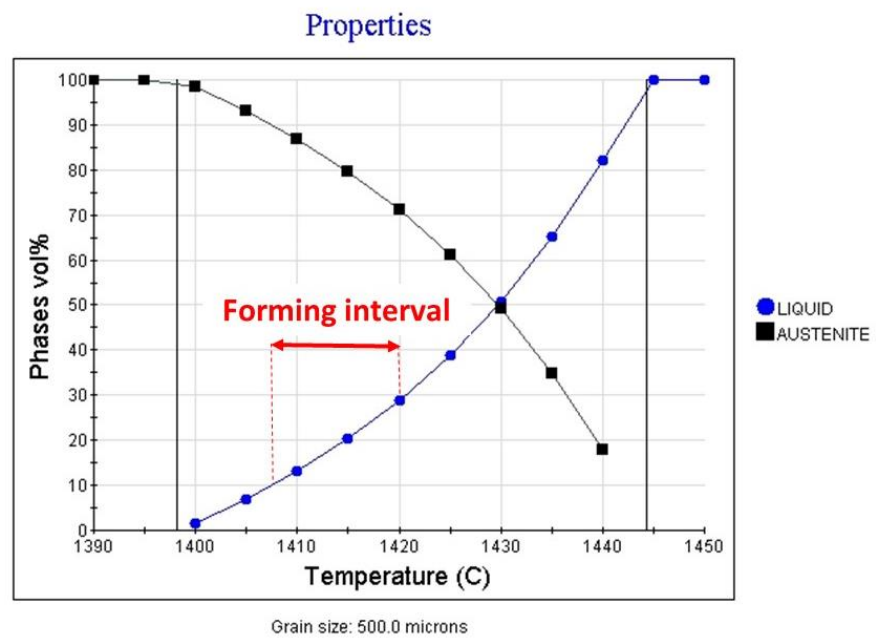

Fig. 1. Calculated feasible forming interval 
The experiments were carried out in special mini-thixoforming equipment which had been designed for handling and processing a very small amount of material in partially melted state (Fig. 2). It relies on high-frequency electrical resistance heating for precise temperature control and generation of the required temperature fields in the workpiece [7]. The actual mini-thixoforming operation consisted in lateral extrusion into a mould (Fig. 3).
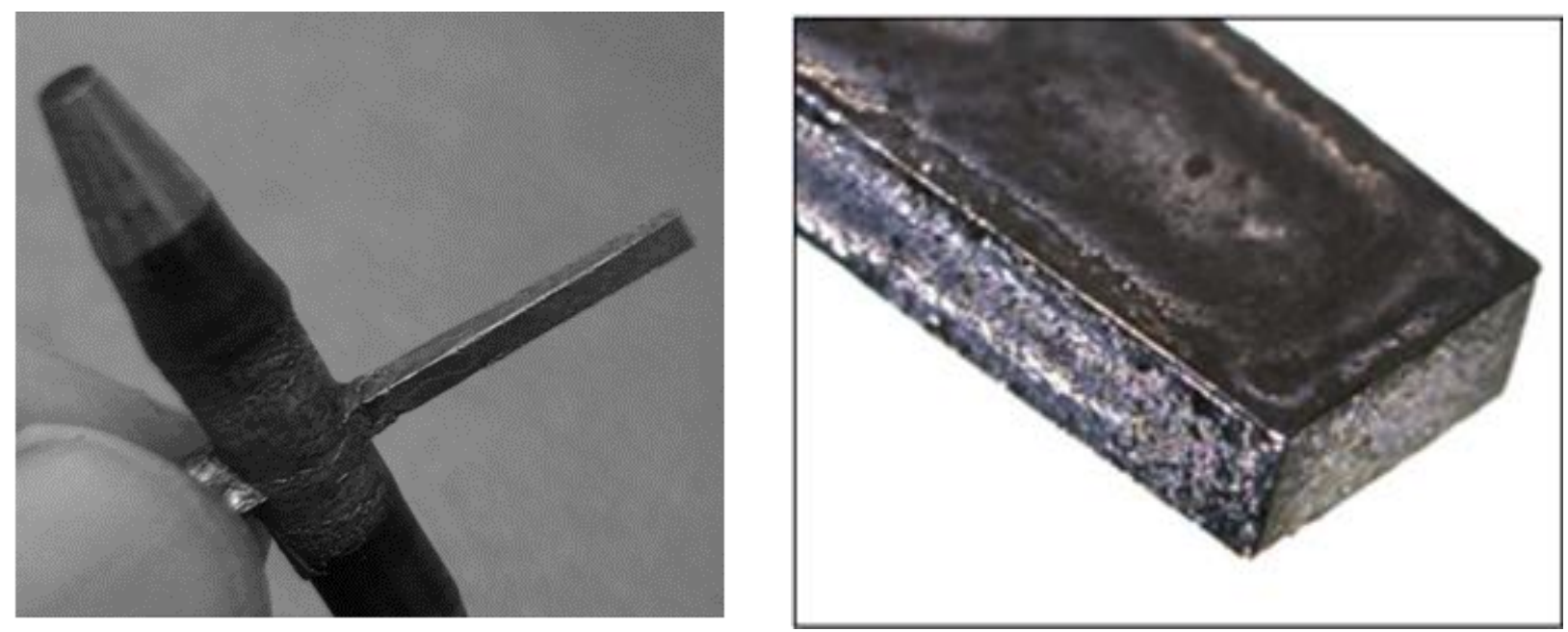

Fig. 2. Typical product manufactured by Mini-thixoforming and the tool in forming machine
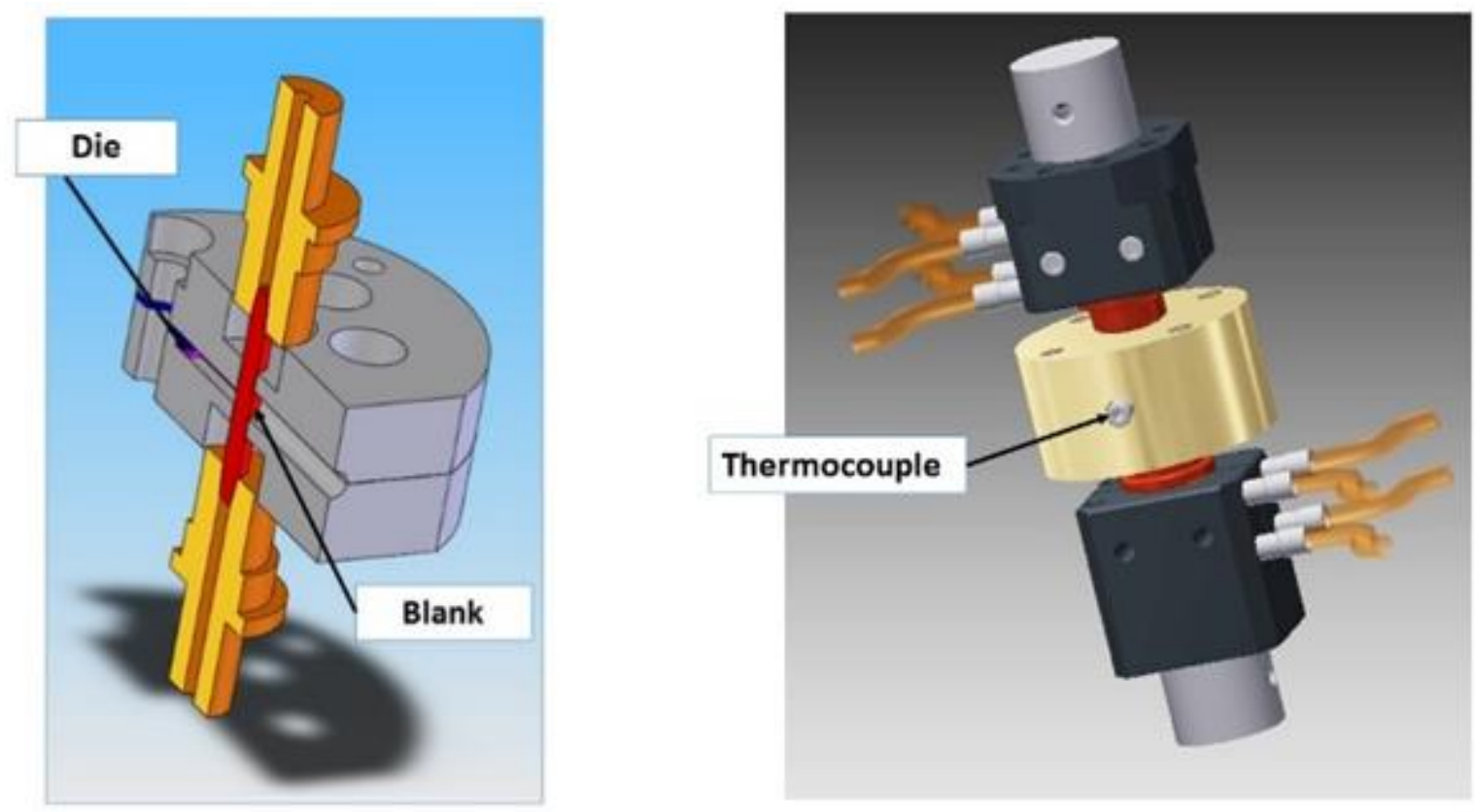

Fig. 3. - Principle of Process

The blanks for the experiment were made of the MS1 Maraging Steel by 3D printing. The total length of the blank was $48 \mathrm{~mm}$, of which the length of the effectively worked part was $38 \mathrm{~mm}$. The diameter was $6 \mathrm{~mm}$. Both ends of the blank were conical with the apex angle of $28^{\circ}$. Their purpose was to centre the blank in the die and provide contact surfaces for energy transfer for heating.

The blanks and the resulting semi-solid processed workpieces were examined under a microscope in both longitudinal and transverse directions. Since the input material for printing was powder, the entire technology chain that governed the microstructural evolution was documented by metallographic techniques.

\section{Discusion of results}

The input material for making the blanks was the commercial powder supplied by the manufacturer of the 3D printing equipment. Its particle size was 5-50 $\mu \mathrm{m}$ (Fig. 4). 

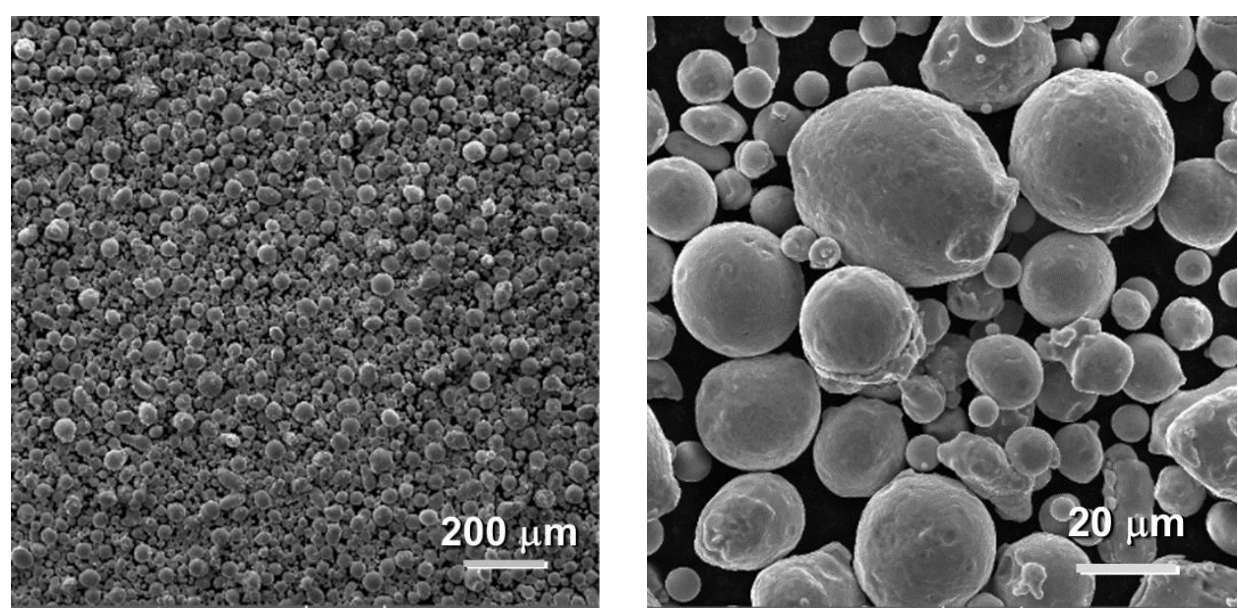

Fig. 4 - Maraging steel powder before printing REM

The 3D printing process was based on laser melting and provided a dense structure of parallel buildup layers with shallow penetration between individual laser paths. In order to acquire excellent properties, maraging steels must be subsequently precipitation-hardened by means of heat treatment. The heat treatment of the buildup therefore provides mainly precipitation of particles of intermediate phases. A major difficulty in the metallographic analysis of these materials is the difference in the etch response of their various states. Nital etching effectively reveals individual laser beam passes and the sharp boundaries between layers of melted metal. In addition, it clearly shows the widths of individual melted regions and the directions of laser passes (Fig. 5a,b). The drawback of this etching reagent is that it inadequately reveals the actual microstructure of the solidified metal. For this reason, an etchant of a mixture of $\mathrm{HCl}$ and $\mathrm{H}_{3} \mathrm{PO}_{4}$ acids (Etchant 2) was used. It effectively reveals the martensitic microstructure of this steel in its non-hardened condition (Fig. $6 a, b)$. At this stage of treatment, the hardness of the workpiece was $360 \mathrm{HV} 10$.

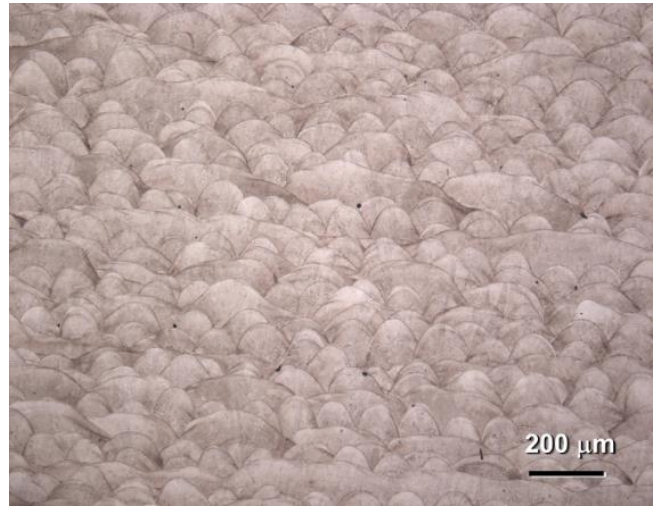

a) Optical microscopy, Nital etch

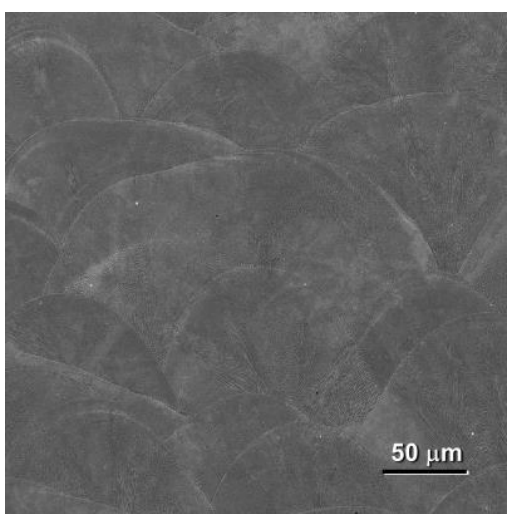

b) Scanning electron micrograph, Nital etch

Fig. 5 - Laser melted microstructure after D3 printing

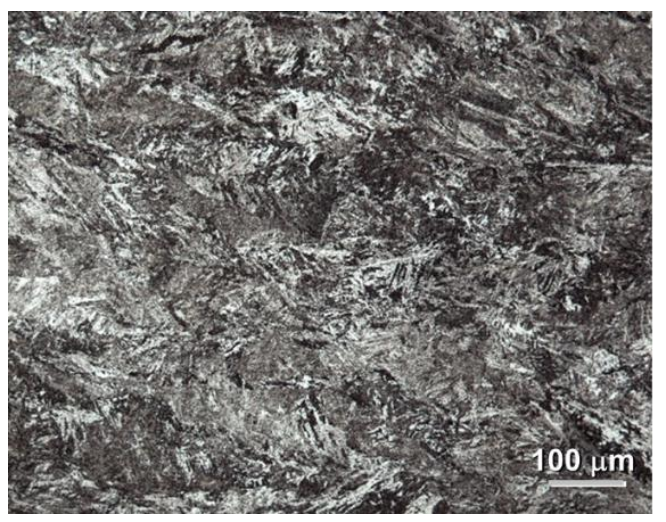

Fig. 6a - Optical micrograph, etched with a mixture of $\mathrm{HCl}$ and $\mathrm{H}_{3} \mathrm{PO}_{4}$ acids

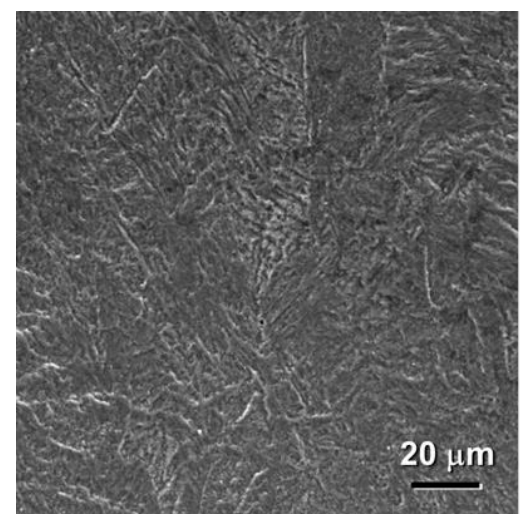

Fig. 6b - REM micrograph, etched with a mixture of $\mathrm{HCl}$ and $\mathrm{H}_{3} \mathrm{PO}_{4}$ acids 
The next operation was stress relieving. As expected, the workpieces showed no considerable microstructural changes, apart from the desired reduction in stress. Considerable changes occurred after the hardening step: uniformly-distributed fine precipitates of intermediate phases have formed in the martensitic structure (Fig. 7a,b).

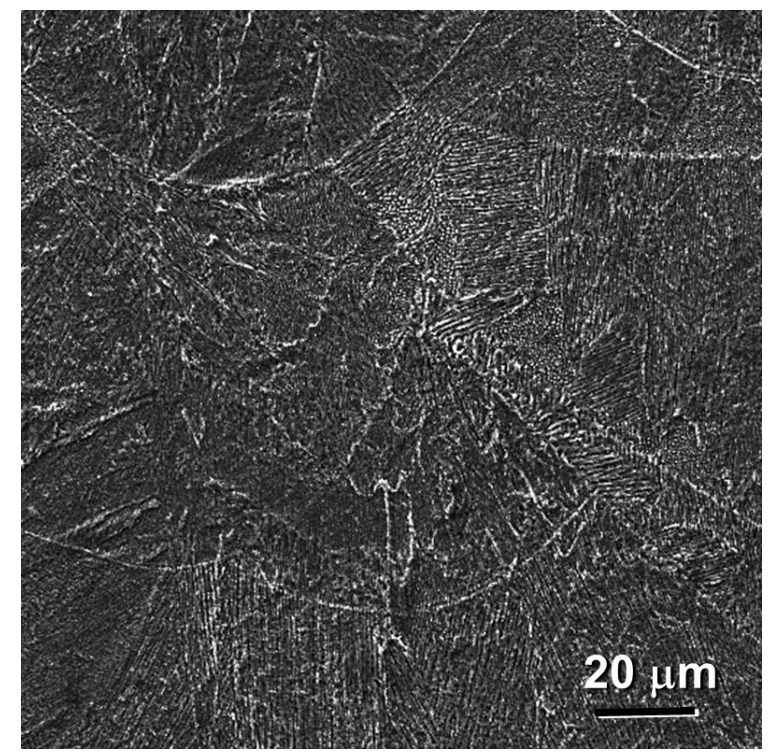

a) melted regions after laser passes, REM

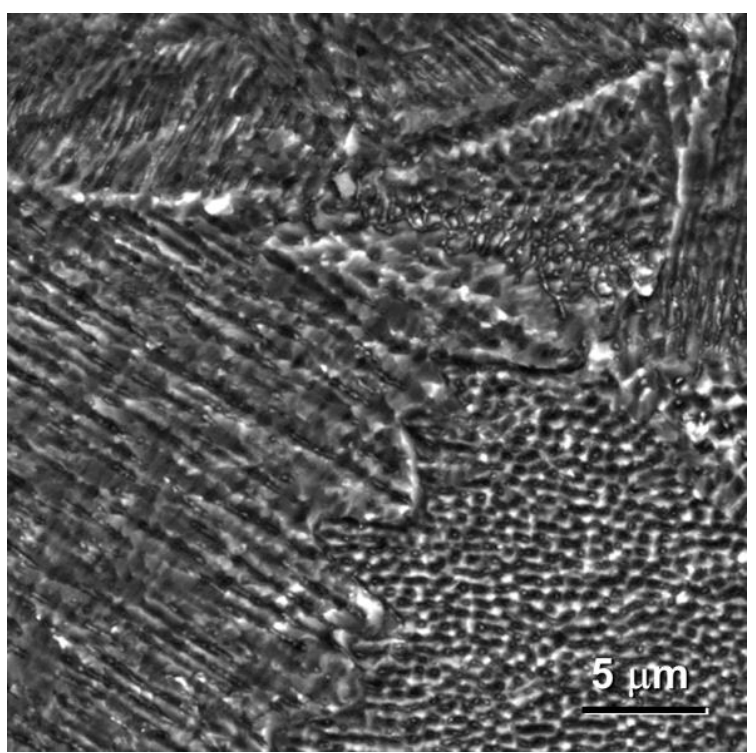

b) precipitates of intermediate phases after hardening detail

Fig. 7 - Microstructure after hardening

The final step of the process chain was mini-thixoforming. It comprised heating to $1440^{\circ} \mathrm{C}$, holding for approximately 10 seconds, and lateral extrusion of the workpiece material into the mould held at room temperature. The heating time was 68 seconds. The forming stroke finished in 0.3 seconds and was followed by rapid solidification thanks to the contact with the surface of the metal die cavity.

An analysis of the product has shown that when appropriate process conditions are used, maraging steel can develop a microstructure that is characteristic of products made by semi-solid processing. Such a structure consists of polyhedral islands of $20-40 \mu \mathrm{m}$ size embedded in a uniform closed network. The fact that it evolves from semi-solid state is confirmed by a characteristic defect which was found by accident in the melted and solidified material between polyhedral islands (Fig. 8). As the region of the semi-solid state available for processing this maraging steel is very narrow, melting also occurred within some of the polyhedral islands. The growth of such melted spots had no immediate constraints which is why they are more or less spherical in shape and have no contact with the network which separates the polyhedral islands. During this process, the thixotropic behaviour was not manifested strongly. Therefore, this material can be considered to be difficult to minithixoform. The next research efforts will focus on the redistribution of chemical elements and on the evolution of microstructural constituents in this process.

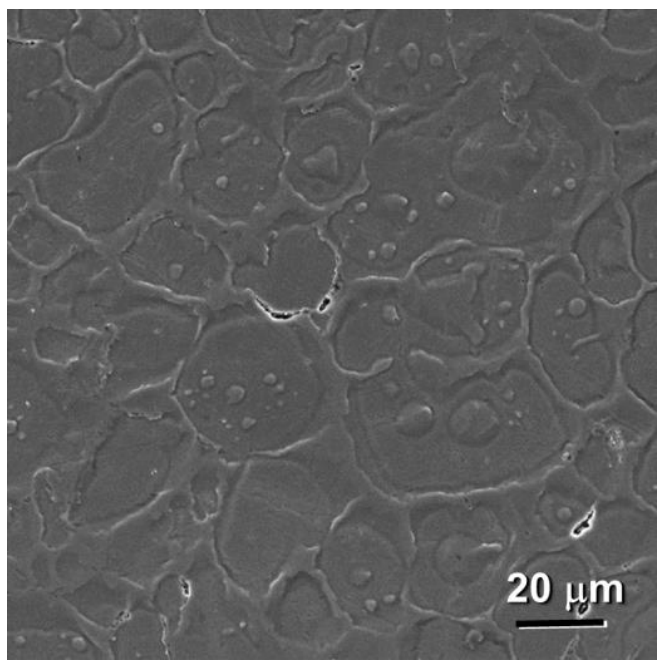

Fig. 8. Micrograph of maraging steel after semisolid processing 


\section{Conclusion}

A maraging steel was chosen for this mini-thixoforming process. The objective was to test a combination of $3 \mathrm{D}$ printing and semi-solid processing. A simple experiment involving lateral extrusion provided information on complex microstructural evolution in the technology chain considered. Suitable and achievable processing parameters have been found which can provide the desired structural state upon semi-solid processing, rapid solidification, and cooling. Given the high processing temperature and the narrow temperature interval, the process became a great technological challenge. Nevertheless, it was completed successfully. Despite that, current findings suggest that at these parameters, the thixotropic property of this material is not favourable. Unless more suitable parameters are found, this material cannot be used in this 3D printing-thixoforming process chain.

\section{Acnowledgements}

This paper presents results achieved under the project LO1502 Development of Regional Technological Institute. The project is subsidised by the Ministry of Education of the Czech Republic from specific resources of the state budget for research and development.

\section{References}

[1] Fleming M.C. Behavior of Metal Alloys in the Semisolid State (1991). Metall Trans A, 22: 957-981.

[2] Thixoforming: Semi-solid Metal Processing. Edited by HIRT G. and KOPP R., pp 1 - 27, Copyright 2009 WILEYVCH Verlag GmbH \& Co. KGaA, Weinheim ISBN: 978-3-527-3220 4-6.

[3] Püttgen W., Bleck W., Hirt G., Shimahara H., Thixoforming of Steels - A Status Report, Advanced Engineering Materiale 9, (2007), No. $4234-245$.

[4] Vančura F., Vorel I., Pileček V., Mašek B. Material Technological Modeling of Thermomechanical Processing of Die Forging of Microalloyed Steel, Kovárenství, 2015, ISSN 1213-9289.

[5] W. H. Bauer and E.A.Collins, in F.R. Eirich (Ed.),Rheology: Theory and Applications, Vol.4, (1967) Academic Press, New York, , ch. 8 .

[6] Wolf, A., Baur, J., Gullo, G.C. Thixoforging, (2012), Available from: http://www.cct-bw.de/veroeffentlichung_pdf/WoBa\%20Massiv01\%20english.pdf.

[7] Ronešová, A., Mašek, B., Staňková, H., Štádler, C. Patent CZ c. 299758 - Method of handling and its shaping at a temperature between solid and liquid, (3. 10. 2008). 